\title{
Targeting the Pedunculopontine Nucleus in Parkinson's disease: Time to Go Back to the Drawing Board
}

\author{
Roger L. Albin ${ }^{1,2,3}$ \\ D. James Surmeier ${ }^{4,5}$ \\ Cecilia Tubert ${ }^{4}$ \\ Martin Sarter ${ }^{3,6}$ \\ Martijn L.T.M. Müller ${ }^{3,7}$ \\ Nicolaas I. Bohnen ${ }^{1,2,3,7}$ \\ William T. Dauer ${ }^{1,2,3,8}$
}

${ }^{1}$ Neurology Service \& GRECC, VAAAHS GRECC, Ann Arbor, MI, 48105, USA

${ }^{2}$ Dept. of Neurology, University of Michigan, Ann Arbor, MI, 48109, USA

${ }^{3}$ University of Michigan Morris K. Udall Center of Excellence for Parkinson’s Disease Research, Ann Arbor, MI, 48109, USA

${ }^{4}$ Dept. of Physiology, Northwestern University, Chicago, IL, 6o611, USA

${ }^{5}$ Northwestern University Morris K. Udall Center of Excellence for Parkinson's Disease Research, Chicago, IL, 6o611, USA

${ }^{6}$ Dept. of Psychology, University of Michigan, Ann Arbor, MI, 48109, USA

${ }^{7}$ Dept of Radiology, University of Michigan, Ann Arbor, MI, 48109, USA

${ }^{8}$ Dept of Cell and Developmental Biology, University of Michigan, Ann Arbor, MI, 48109, USA

This is the author manuscript accepted for publication and has undergone full peer review but has not been through the copyediting, typesetting, pagination and proofreading process, which may lead to differences between this version and the Version of Record. Please cite this article as doi: $10.1002 / \mathrm{mds} .27540$

This article is protected by copyright. All rights reserved. 
Address Correspondence to: Roger L. Albin, MD, 5023 BSRB, 109 Zina Pitcher Place, Ann Arbor, MI, 48109-2200, USA; (ph) 734-764-1347; (fax) 734-763-7686; ralbin@umich.edu Text Word Count: 2259

Running Title: Reconsider PPN DBS

Keywords: Deep Brain Stimulation, Acetylcholine, Dopamine, Substantia Nigra

Financial Disclosure-Conflicts: The authors have no relevant financial disclosures or conflicts of interest.

Funding Sources: Supported by $\mathrm{P}_{50}$ NSo91856, R21 NSo88302, and the Michael J. Fox Foundation. 
Subthalamic or pallidal Deep Brain Stimulation (DBS) are effective treatments for selected Parkinson disease (PD) patients. The effectiveness of subthalamic nucleus (STN) or pallidal DBS led logically to efforts to employ DBS in other brain regions to treat aspects of PD lacking adequate medical treatments. Gait and balance deficits unresponsive to dopamine replacement therapy are major problems in more advanced PD patients. The absence of satisfactory medical treatments for these problems led to human clinical experiments deploying DBS in the region of the pedunculopontine nucleus (PPN) with the goal of alleviating crippling gait and balance problems. The fundamental rationale was that low frequency DBS in the PPN would increase the activity of a critical group of PPN neurons. The clinical experience with PPN DBS was reviewed recently'. In this Viewpoint, we discuss the scientific rationale for PPN DBS and argue that our present understanding of the PPN, surrounding regions, and their potential roles in locomotion does not provide a good scientific rationale for conventional PPN DBS in PD.

\section{Original Rationale for PPN DBS}

The origins of the scientific rationale for PPN DBS lies in the 1960's when investigators in the Soviet Union discovered that the region of the mesopontine junction is important in controlling locomotion and posture. These investigators found that low intensity electrical stimulation in this region induced locomotion in decerebrate cats. ${ }^{2}$ This electrophysiologically defined region was called the Mesencephalic Locomotor Region (MLR). Subsequent work by Garcia-Rill and colleagues suggested that the MLR is centered around the PPN, which is populated by cholinergic neurons. ${ }^{3,4}$ Garcia-Rill and colleagues suggested a model in which MLR-PPN neurons projected to a region of the ventromedial medulla (VMM) connected to spinal central pattern generators 
responsible for locomotion. ${ }^{4}$ Because cholinergic neurons were a prominent part of the PPN, it was widely inferred that they were critical mediators of forebrain inputs regulating locomotion, though retrograde tracing data from Garcia-Rill and colleagues strongly implicated noncholinergic PPN neurons as the major source of PPN to VMM projections. ${ }^{5}$

The PPN is a heterogeneous region composed of cholinergic, glutamatergic, GABAergic, and probably glycinergic neurons. ${ }^{6,7,8}$ These different neuron types are not distributed uniformly within the PPN. Cholinergic neurons are denser in the caudal than in the rostral portion of the PPN, glutamatergic neuron density increases along the rostral to caudal axis, while the GABAergic neurons show the opposite pattern. ${ }^{6,8}$ The connectome of these populations is heterogeneous. Oakman and coworkers ${ }^{9}$, for example, found that substantia nigra pars compacta (SNc) neurons receive cholinergic innervation mainly from the rostroventral and medial PPN, while ventral tegmental area (VTA) neurons receive cholinergic inputs principally from caudal PPN. The cytological and connectional heterogeneity of the PPN is in marked contrast to relatively homogeneous STN and GPi. This has potential implications for PPN DBS as a variation in DBS electrode position is likely to modulate different circuitry with differing effects on motor behavior.

Several observations subsequently suggested that PPN cholinergic neurons were involved in gait and balance problems in PD and related disorders. Hirsch et al. described loss of cholinergic PPN neurons in PD and Progressive Supranuclear Palsy. ${ }^{10}$ The magnitude of cholinergic PPN neuron 
loss was described as greater in PD subjects with a history of falls, consistent with a critical role for cholinergic PPN neurons in gait and balance control. ${ }^{11}$ Positron emission tomography imaging studies of cholinergic terminal integrity performed by some of us (NIB, MM, RLA) supported this inference. ${ }^{12,13,14}$ Subsequent work, however (see below), showed that there also is loss (albeit not as great) of other PPN neuronal populations in PD patients.

Karachi et al. moved beyond correlative studies with experiments in non-human primates to assess whether loss of PPN cholinergic neurons could be causally linked to gait and posture deficits. First, they showed that lesioning cholinergic PPN neurons caused gait, balance, and posture deficits. Second, they showed that with MPTP lesions, loss of PPN cholinergic neurons exacerbated dopamine insensitive gait, balance, and posture deficits ${ }^{11,15}$. More recently, pharmacogenetic stimulation of PPN cholinergic neurons was reported to reverse gait and postural abnormalities in a rat toxin PD model. ${ }^{16}$

In addition, some studies suggested the emergence of pathophysiological PPN activity in rodent PD toxin models. ${ }^{17,18,19}$ Breit et al. described increased activity and bursting of PPN neurons after 6-OHDA lesions. Although it was unclear which PPN neuron subpopoulations was contributing to the pathophysiology, it established a parallel with the pathophysiology observed in STN of PD patients and the hypothesis that DBS might ameliorate symptoms. Breit et al. also described relative normalization of PPN neuron activity in animals with joint 6-OHDA SNc lesions and ibotenic acid STN lesions. ${ }^{18}$ The PPN receives a number of afferent projections from the basal 
ganglia, ${ }^{6,7}$ and it is plausible that basal ganglia dysfunction would result in PPN-MLR dysfunction, providing an additional rationale for DBS targeting of the PPN in PD.

\section{Recent Studies Contradicting the Original Rationale}

Results of other experiments, however, contradict the idea that cholinergic PPN neurons are critical for gait. Neither indiscriminate excitotoxic lesions of PPN neurons nor selective lesions of cholinergic PPN neurons produced marked abnormalities in gait in rodents. Selective lesions of PPN cholinergic neurons did not worsen gait impairments resulting from basal forebrain cholinergic and striatal dopamine lesions. ${ }^{20,21}$.

In part, discrepant results may be a consequence of the poorly defined anatomy of the operationally-defined MLR. As mentioned above, the MLR was defined on the basis of the motoric response to electrical stimulation - an approach that alone cannot be used to precisely define the somatodendritic location of the obligate neurons. Work by Takakusaki in decerebrate cats suggests that the cuneiform nucleus, which is rostral to the PPN, is essential for stimulationinduced locomotion $^{22}$. Lu and colleagues' experiments in rats have implicated a group of neurons ventral to the PPN that project directly to the spinal cord pattern generators. ${ }^{23,24}$ In fact, work with newer tools or tool combinations suggests that the key node for locomotion may not be the PPN. 
Studies using optogenetic methods to selectively activate neuron subpopulations within the MLR shed further doubt on the centrality of PPN cholinergic neurons for locomotion and highlight the complexity of MLR functional anatomy. Roseberry et al. attempted to stimulate cholinergic neurons, GABAergic neurons, and glutamatergic neurons in this region. ${ }^{25}$ For technical reasons, it was difficult to stimulate cholinergic neurons adequately. In resting mice, stimulation of glutamatergic neurons elicited locomotion. In locomoting mice, stimulation of glutamatergic PPN neurons significantly accelerated locomotion and stimulation of GABAergic PPN neurons slowed locomotion. This work is consistent with a role for the PPN in the MLR but suggest that cholinergic PPN neurons are not the critical population for locomotion. These results are consistent with the older Garcia-Rill group model. In older tract-tracing studies, the PPN-VMM projection identified as the key descending pathway for gait activation was found to consist mainly of non-cholinergic neurons. Also consistent with the MLR to VMM model, Capelli et al. recently described caudal brainstem circuits controlling locomotor velocity. ${ }^{26}$ This group traced MLR efferents to the caudal medulla and found that they originated in both PPN and cuneiform nuclei glutamatergic neurons. Caggiano et al. describe both PPN and cuneiform nucleus glutamatergic neurons as important for gait control in mice with complex interactions (including direct interconnections) between these two nuclei influencing gait speed and gait modes. ${ }^{27}$

While the original conception of the role of cholinergic PPN neurons focused on regulation of brainstem control of locomotion, these neurons have a major rostral projection to the thalamus, suggesting a role in modulation of sensory information. ${ }^{6,7}$ This concept is supported by research 
in PD subjects indicating a role for these projections in sensory integration to maintain normal posture and detection of salient external cues. ${ }^{28,29}$

PPN glutamatergic neurons also project rostrally. A recent study demonstrated that PPN glutamatergic neurons robustly activate SNc dopaminergic neurons, creating yet another way in which these neurons might initiate movement. ${ }^{30}$ By comparison, optogenetic activation of PPN cholinergic neurons evokes much smaller responses that are weakly linked to changes in SNc DA neuron spiking. ${ }^{31}$

\section{Lessons from Human Studies}

These results may explain data from some human PPN DBS studies. Two studies report significant improvement in gait parameters with PPN DBS in the OFF medication state, but not in the ON medication state. ${ }^{32,33}$ It is plausible that in the OFF medication state, PPN activation of dopaminergic neurons produces enough incremental striatal dopamine to make a difference in locomotion. In the $\mathrm{ON}$ medication state, however, this modest effect may be obscured by the effects of dopamine replacement treatment. In contrast, Stefani and coworkers found that PPN DBS stimulation improved gait and posture both in OFF and ON states. ${ }^{34}$ Further clouding interpretation of the clinical outcome data, some PD subjects receiving PPN DBS also underwent DBS at conventional targets such as the STN. ${ }^{1}$ In their recent review, Thevathasan et al. conclude that despite the variation in outcomes, when properly positioned, PPN DBS has the potential to reduce falls and improve gait in PD patients. ${ }^{1}$ Thevathasan et al. are appropriately cautious about 
the existing clinical outcome data and stress that the effect of PPN DBS on the quality of life of PD patients remains unproven. ${ }^{1}$ Efforts at meta-analysis of individual subject data support these conclusions..$^{35-37}$

In spite of some beneficial outcomes in PD patients, the uncertainties surrounding the nature of the MLR and the neuronal heterogeneity of the mesopontine region pose significant theoretical obstacles to the use of PPN DBS. Is the PPN the appropriate target? Given the small sizes of the involved nuclei and their considerable heterogeneity, the electric fields generated by standard DBS electrodes are likely to modulate the activity of multiple nuclei at the mesopontine junction. ${ }^{3}$ In their pioneering studies of rostral human brainstem cholinergic neurons, Mesulam et al. emphasize that the distribution of mesopontine cholinergic neurons does not follow clear nuclear boundaries and that their dendrites interpenetrate a number of surrounding structures. ${ }^{38}$ This is likely true for other PPN neuronal populations as well. As shown by the optogenetic experiments, there is considerable functional heterogeneity within this region. The mesopontine region is characterized also by the presence of a number of important white matter tracts, increasing the risk of significant side effects of stimulation. The challenges of developing accurate stereotaxic targeting algorithms and correlating electrode placement, clinical outcomes, and underlying anatomy in the rostral brainstem underscores these concerns. ${ }^{39}$ Even if DBS electrode actions could be restricted to one of these nuclei, different and likely contradictory effects will result from indiscriminate effects of conventional DBS. Consistent with this concern, rats with combined 
toxin lesions of the substantia nigra and PPN were reported to exhibit worsening gait performance upon receiving a PPN DBS analogue..$^{20}$

In addition, there is conflicting information about the effects of dopamine depletion on PPN neuronal activity. In non-human primates and in PD patients, data suggest that the PPN activity is suppressed in PD; pharmacological disinhibition improves motor behavior in parkinsonian MPTP-lesioned primates. ${ }^{40}$ In contrast, rodent studies suggest either PPN activity is elevated ${ }^{18,41,42}$, suppressed $^{41,43}$, or not changed ${ }^{45,46}$ after dopamine depletion. A recent, small non-human primate study did not find a change in PPN activity in MPTP lesioned non-human primates. ${ }^{47}$ These discrepancies have yet to be resolved but are likely to stem from the heterogeneity in cell types and circuit complexity.

Another issue is that many PPN neurons degenerate in PD. This includes not only cholinergic PPN neurons, but also GABAergic and glycinergic neurons.$^{48}$ How this pathology affects the response of neurons to electrical stimulation is unclear. If the goal is to boost the activity of PPN neurons that are compromised, then low frequency stimulation, near the normal autonomous rate of cholinergic PPN neurons, should be more effective than higher frequency stimulation which is likely to drive neurons into depolarization block. Indeed, low frequency - but not high frequency - PPN stimulation has been reported to improve akinesia in parkinsonian monkeys. ${ }^{49}$

\section{Summary}

This article is protected by copyright. All rights reserved. 
In summary, what we have learned about the PPN and MLR over the past few years undermines the simplistic model that underpins the rationale for PPN DBS. Our understanding of the relevant circuitry remains rudimentary. Further, much of what we know about these circuits is based on studies in rodents and felines. While it is likely that critical features of posture and locomotion control are phylogenetically conserved, additional non-human primate experiments are needed. Even if we understood the relevant motor circuits in detail, the small sizes of these structures, and the considerable heterogeneity of neurons within the PPN and surrounding structures, indicates that conventional DBS is too blunt an instrument to selectively target the relevant neurons. Finally, manipulating sick neurons adds an additional element of uncertainty. In the context of these facts, it is not surprising that the clinical results of PPN DBS are largely unimpressive. ${ }^{1}$ Technical refinements in conventional DBS targeting or technology are unlikely to overcome these obstacles.

While basic research revealed significant obstacles to manipulating mesopontine neuronal populations, it also indicates circuits in this region are important in gait and balance, and likely relevant to PD. It is plausible that cell type specific manipulations in this region with optogenetic or chemogenetic methods might be useful. ${ }^{\circ}$ That said, deploying these technologies in welldesigned clinical experiments face a number of hurdles, not the least of which is a much firmer grasp of the neural circuitry controlling gait and posture is required. We need also as well to know how this circuitry is disrupted in PD..$^{51}$ At this point in time, human experimentation with PPN DBS should be reconsidered. Efforts should focus on sorting out precisely how the 
PPN/MLR and their afferent and efferent connections work, on what dysfunctions are characteristic of $\mathrm{PD}$, and on developing the technologies needed to rectify these dysfunctions in PD patients. 
Author Roles: RLA, WTD, and DJS conceived this article. RLA prepared the initial draft. RLA, DJS, CT, WTD, NIB, MTLMM, and MS all participated in critical revisions and preparation of the final version.

\section{Financial Disclosures:}

RLA receives compensation of DSMB service for the LEGATO-HD (Teva/ICON), HTT $_{\mathrm{RX}}$ (Ionis), and ${ }_{251} \mathrm{PP}_{301}$ (Biogen/ICON) trials. He receives grant support from R25 NSo8945o, R21

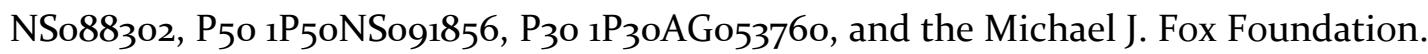
DJS received compensation for consulting services Adamas Pharmaceuticals and Pfizer, Inc. He has grant support from NIH, CHDI, MJFF, JPB Foundation, Bumpus Foundation, IDP Foundation, NPF, Adamas Pharmaceuticals, and John Flanagan.

CT has nothing to disclose.

MS received compensation for consulting services for Takeda Pharmaceuticals Inc. and Pfizer Inc. He receives grant support from H. Lundbeck (Denmark), Takeda Pharmaceuticals Inc., and PHS Grants $\mathrm{P}_{50 N S o 918} 56$ and PO1 DAo31656.

NIB receives grant support from the NIH, Michael J. Fox Foundation, the Department of Veteran Affairs, Chase Pharmaceuticals and Axovant Sciences.

MLTMM receives grant support from $\mathrm{P}_{50 N S o 918} 6$ and the Michael J. Fox Foundation.

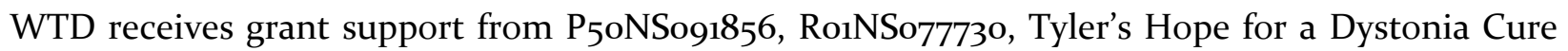
Foundation, and the Michael J. Fox Foundation 


\section{References:}

1) Thevathasan W, Debu B, Aziz T, et al. Pedunculopontine nucleus deep brain stimulation in Parkinson's disease: A clinical review. Mov Disord 2018:33:10-20.

2) Shik ML, Severin FV, Orlovskii GN. Control of walking and running by means of electrical stimulation of the midbrain. Biofizika 1966;11:659-666.

3) Garcia-Rill E, Hyde J, Kezunovic N, Urbano FJ, Petersen E. The physiology of the pedunculopontine nucleus: implications for deep brain stimulation. J Neural Transm 2015;122:225-235.

4) Garcia-Rill E. The pedunculopontine nucleus. Prog Neurobiol 1991;36:363-389.

5) Skinner RD, Kinjo N, Ishikawa Y, Biedermann JA, Garcia-Rill E. Locomotor projections from the pedunculopontine nucleus to the medioventral medulla. Neuroreport 1990;1:207-210.

6) Mena-Segovia J, Bolam JP. Rethinking the Pedunculopontine Nucleus: From Cellular Organization to Function. Neuron 2017;95:7-18.

7) Pienaar I, Vernon A, Winn P. The Cellular Diversity of the Pedunculopontine Nucleus: Relevance to Behavior in Health and Aspects of Parkinson's Disease. The Neuroscientist 2016;pii:1073858416682471. [Epub ahead of print]

8) Wang HL, Morales M. Pedunculopontine and laterodorsal tegmental nuclei contain distinct populations of cholinergic, glutamatergic and GABAergic neurons in the rat. Eur J Neurosci 2009;29:340-358.

This article is protected by copyright. All rights reserved. 
9) Oakman SA, Faris PL, Kerr PE, Cozzari C, Hartman BK. Distribution of pontomesencephalic cholinergic neurons projecting to substantia nigra differs significantly from those projecting to ventral tegmental area. J Neurosci 1995;15:5859-5869.

10) Hirsch EC, Graybiel AM, Duyckaerts C, Javoy-Agid F. Neuronal loss in the pedunculopontine tegmental nucleus in Parkinson disease and in progressive supranuclear palsy. Proc Natl Acad Sci U S A 1987;84:5976-5980.

11) Karachi C, Grabli D, Bernard FA, et al. Cholinergic mesencephalic neurons are involved in gait and postural disorders in Parkinson disease. J Clin Invest 2010;120:2745-2754.

12) Müller ML, Albin RL, Kotagal V, Koeppe RA, Scott PJ, Frey KA, Bohnen NI. Thalamic cholinergic innervation and postural sensory integration function in Parkinson's disease. Brain 2013;136:3282-3289.

13) Bohnen NI, Müller ML, Kotagal V, Koeppe RA, Kilbourn MR, Gilman S, Albin RL, Frey KA. Heterogeneity of cholinergic denervation in Parkinson's disease without dementia.J Cereb Blood Flow Metab 2012;32:1609-1617.

14) Bohnen NI, Müller ML, Koeppe RA, Studenski SA, Kilbourn MA, Frey KA, Albin RL. History of falls in Parkinson disease is associated with reduced cholinergic activity. Neurology 2009;73:1670-1676.

15) Grabli D, Karachi C, Folgoas E, et al. Gait disorders in parkinsonian monkeys with pedunculopontine nucleus lesions: a tale of two systems. J Neurosci 2013;33:11986-11993.

This article is protected by copyright. All rights reserved. 
16) Pienaar IS, Gartside SE, Sharma P, et al. Pharmacogenetic stimulation of cholinergic pedunculopontine neurons reverses motor deficits in a rat model of Parkinson's disease. . Mol Neurodegener 2015;10:47. doi: 10.1186/s13024-015-0044-5.

17) Carlson JD, Pearlstein RD, Buchholz J, Iacono RP, Maeda G. Regional metabolic changes in the pedunculopontine nucleus of unilateral 6-hydroxydopamine Parkinson's model rats. Brain Res 1999;828:12-19.

18) Breit S, Bouali-Benazzouz R, Benabid AL, Benazzouz A. Unilateral lesion of the nigrostriatal pathway induces an increase of neuronal activity of the pedunculopontine nucleus, which is reversed by the lesion of the subthalamic nucleus in the rat. Eur J Neurosci 2001;14:1833-1842.

19) Orieux G, Francois C, Féger J, et al. Metabolic activity of excitatory parafascicular and pedunculopontine inputs to the subthalamic nucleus in a rat model of Parkinson's disease. Neuroscience 2000;97:79-88.

20) Gut NK, Winn P.Deep brain stimulation of different pedunculopontine targets in a novel rodent model of parkinsonism. J Neurosci 2015;35:4792-4803.

21) Kucinski A, Sarter M. Modeling Parkinson's disease falls associated with brainstem cholinergic systems decline. Behav Neurosci 129:96-104.

22) Takakusaki K. Functional Neuroanatomy for Posture and Gait Control. J Mov Disord 2017;10:1-17.

23) Thankachan S, Fuller PM, Lu J. Movement- and behavioral state-dependent activity of pontine reticulospinal neurons. Neurosci 2012;221:125-139.

This article is protected by copyright. All rights reserved. 
24) Sherman D, Fuller PM, Marcus J, et al. Anatomical localization of the mesencephalic locomotor region and its possible role in locomotion, posture, cataplexy, and Parkinsonism. Front Neurol 2015;6:140. doi: 10.3389/fneur.2015.00140.

25) Roseberry TK, Lee AM, Lalive AL, Wilbrecht L, Bonci A, Kreitzer AC. Cell-Type-Specific Control of Brainstem Locomotor Circuits by Basal Ganglia. Cell 2016;164:526-537.

26) Capelli P, Pivetta C, Esposito MS, Arber S. Locomotor speed control circuits in the caudal brainstem. Nature 2017;551:373-377.

27) Caggiano V, Leiras R, Goni-Erro H, et al. Midbrain circuits that set locomotor speed and gait selection. Nature 2018;553:455-46o.

28) Muller ML, Albin RL, Kotagal V, et al. Thalamic cholinergic innervation and postural sensory integration function in Parkinson's disease. Brain 2013;136:3282-3289.

29) Kim K, Müller MLTM, Bohnen NI, Sarter M, Lustig C. Thalamic cholinergic innervation makes a specific bottom-up contribution to signal detection: Evidence from Parkinson's disease patients with defined cholinergic losses. Neuroimage;2017:149:295-204.

30) Galtieri DJ, Estep CM, Wokosin DL, Traynelis S, Surmeier DJ. Pedunculopontine glutamatergic neurons control spike patterning in substantia nigra dopaminergic neurons. eLife 2017; 6:e30352.

31) Xiao C, Cho JR, Zhou C, et al. Cholinergic mesopontine signals govern locomotion and reward through dissociable midbrain pathways. Neuron 2016;90:333-347.

32) Thevathasan W, Cole MH, Greapel CL, et al. A spatiotemporal analysis of gait freezing and the impact of pedunculopontine nucleus stimulation. Brain 2012;135:1446-1454.

This article is protected by copyright. All rights reserved. 
33) Ferraye MU, Debu B, Fraix L, et al. Effects of pedunculopontine nucleus area stimulation on gait disorders in Parkinson's disease. Brain 2010;133:205-214.

34) Stefani A, Lozano AM, Peppe A, et al. Bilateral deep brain stimulation of the pedunculopontine and subtalamic nuclei in severe Parkinson's disease. Brain 2007;130:1596-1607.

35) Wang J-W, Zheng Y-Q, Zhang X-H, Wang Y-P, Li J-P, Li Y-L. Deep brain stimulation of pedunculopontine nucleus for postural instability and gait disorder after Parkinson disease: a meta-analysis of individual patient data. World Neurosurg 2017;102:72-78.

36) Wang H, Gao H, Jiao T, Luo Z. A meta-analysis of the pedunculopontine nucleus deepbrain stimulation effects on Parkinson's disease. Neuroreport 2016;27:1336-1344.

37) Golestanirad L, Elahi B, Graham SJ, Das S, Wald LL. Efficacy and safety of pedunculopontine nuclei (PPN) deep brain stimulation in the treatment of gait disorders: a meta-analysis of clinical studies. Can J Neurol Sci 2016;43:120-126.

38) Mesulam MM, Geula G, Bothwell MA, Hersh LB. Human reticular formation: cholinergic neurons of the pedunculopontine and laterodorsal tegmental nuclei and some cytochemical comparisons with forebrain cholinergic neurons. J Comp Neurol 1988;281:611-633.

39) Goetz L, Bhattacharjee M, Ferraye MU, et al. Deep brain stimulation of the pedunculopontine nucleus area in Parkinson disease: MRI-based anatomoclinical correlations and optimal target. Neurosurg 2018;doi: 10.1093/neuros/nyy151. [Epub ahead of print] 
40) Nandi D, Aziz TZ, Giladi N, Winter J, Stein JF. Reversal of akinesia in experimental parkinsonism by GABA antagonist microinjections in the pedunculopontine nucleus. Brain 2002;125:2418-2430.

41) Breit S, Lessmann L, Benazzouz A, Schulz JB. Unilateral lesion of the nigrostriatal pathway induces hyperactivity in the subthalamic nucleus and subtantia nigra in the rat. Eur J Neurosci 2005;22:2283-2294.

42) Jeon MF, Ha y, Cho YH, Lee BH, Park YG, Chang JW. Effect of ipsilateral subthalamic nucleus lesioning in a rat parkinsonian model: study of behavior correlated with neuronal activity in the pedunculopontine nucleus. J Neurosurg 2003;99:762-767.

43) Fiorio T, Scarnati E, Confalone G, et al. High-frequency stimulation of the subthalamic nucleus modulates the activity of pedunculopontine neurons through direct activation of excitatory fibres as well as through indirect activation of inhibitory pallidal fibres in the rat. Eur J Neurosci 2007;25:1174-1186.

44) Gomez-Gallego M, Fernandez-Villalba E, Fernandez-Barreiro A, Herrero MT. Changes in the neuronal activity in the pedunculopontine nucleus in chronic MPTP-treated primates: an in situ hybridization study of cytochrome oxidase subunit I, choline acetyl transferase and substance P mRNA expression. J Neural Transm 2007;114:319-326.

45) Mena-Segovia J, Ross HM, Magill PJ, Bolam JP. The pedunculopontine nucleus: towards a functional integration with the basal ganglia. In: Bolam JP, Ingham CA, Magill PJ (Eds.). The Basal Ganglia VIII 2005. Springer Science+Business Media, Inc, New York, 523-532. 
46) Heise CE, Mitrofanis J. Fos immunoreactivity in some locomotor neural centers of 6OHDA-lesioned rats. Anar Embryol (Berl) 2006;211:659-671.

47) Goetz L, Piallat B, Bhattacharjee M, Mathieu H, David O, Chabardès S. Spike discharge characteristic of the caudal mesencephalic reticular formation and pedunculopontine nucleus in MPTP-induced primate model of Parkinson disease. Neurobiol Dis 2018;pii: So969-9961(18)30394-2. doi: 10.1016/j.nbd.2018.08.002

48) Pienaar IS, Elson JL, Racca C, Nelson G, Turnbull DM, Morris CM. Mitochondrial abnormality associates with type-specific neuronal loss and cell morphology changes in the pedunculopontine nucleus in Parkinson disease. Am J Pathol 2013;183:1826-1840.

49) Jenkinson N, Nandi D, Miall RC, Stein JF, Aziz TZ. Pedunculopontine nucleus stimulation improves akinesia in a parkinsonian monkey. NeuroReport 2004; 15-2621-2624.

5o) Glavan A, Stauffer WR, Acker L., et al. Nonhuman primate optogenetics: Recent Advances and future directions. J Neurosci 2017;37:10894-10903.

51) Goetz L., Piallat B, Bhattarcharjee M, et al. On the role of the pedunculopontine nucleus and mesencephalic reticular formation in locomotion in nonhuman primates. J Neurosci 2016;36:4917-4929.

This article is protected by copyright. All rights reserved. 